\title{
ESTUDIO DE LOS ACONTECIMIENTOS METEOROLÓGICOS EXTRAORDINARIOS EN LA COMUNIDAD AUTÓNOMA DEL PAÍS VASCO (1870-1954) A TRAVÉS DE LA PRENSA*
}

\author{
Laura Hernández Varela \\ Miguel Ángel Lozano Valencia \\ Carolina Soleto García \\ Departamento de Geografía, Prehistoria y Arqueología \\ Universidad del País Vasco
}

\section{RESUMEN}

El artículo expone los resultados y la metodología utilizada para el análisis de varios acontecimientos climáticos extraordinarios, mediante la utilización de la información obtenida a través de la prensa, referente a la C.A.P.V. en el intervalo 1870-1954. El estudio revela dos periodos significativos, de 1895 a 1917 y de 1924 a 1935. Las provincias litorales, en especial Gipuzkoa, son las que mayor número de acontecimientos registran, destacando las nevadas extraordinarias y los fenómenos relacionados con las fuertes precipitaciones (aguaceros, temporales de lluvia, crecidas e inundaciones).

Palabras clave: acontecimiento climático extraordinario, noticia, C.A.P.V.

\section{ABSTRACT}

The article shows the results and the methodology used for the analysis of some extraordinary climatic events, using information of years 1870 to 1954 from C.A.P.V.'s newspapers. The study shows two significative periods, from 1895 to 1917 and from 1924 to 1935 . The coastal provinces, especially Gipuzkoa, undergo maximun number of events, highlight extraordinary snowfalls and other phenomenas related to heavy rain (heavy showers, storms, spates and floods).

Key Words: extraordinary climatic events, news, C.A.P.V.

\footnotetext{
* Este artículo es resultado del trabajo «Acontecimientos meteorológicos extraordinarios en la C.A.P.V. (1870-1954)» que se ha elaborado dentro del proyecto «Fuentes para el estudio del clima del País Vasco. Organización de un sistema documental» dirigido por Eugenio Ruiz Urrestarazu que se está llevando a cabo por el Departamento de Geografía, Prehistoria y Arqueología de la U.P.V./E.H.U, financiado por la Dirección de Climatología y Meteorología del Dpto. de Transportes y Obras Públicas del Gobierno Vasco.
} 


\section{Introducción}

El estudio de acontecimientos climáticos extraordinarios goza de larga tradición dentro de la Climatología de nuestro país. En los últimos años, se ha producido un aumento del interés de estos temas debido a los daños ocasionados en las actividades humanas por estos eventos, con el objetivo de aplicar medidas preventivas.

El análisis de riesgos climáticos en España ha sido abordado desde diferentes disciplinas, como la Climatología, la Meteorología y la Economía.

La comunidad geográfica española, se ha centrado en la realización de estudios sobre episodios específicos. El primer antecedente de este tipo de trabajos lo podemos encontrar a mediados de los años 50 en la revista Estudios Geográficos, correspondiente al profesor López Gómez. Se trata de un artículo sobre el episodio de helada de 1956 que produjo importantes daños en la agricultura valenciana. A partir de aquí se sucedieron estudios sobre crecidas, sequías y catastróficas inundaciones.

En cuanto a los primeros trabajos elaborados por meteorólogos cabe mencionar los Resúmenes de observaciones meteorológicas anuales en las primeras décadas del siglo XX. A estos estudios, le acompañaron algunas obras, entre las que cabe destacar los trabajos de Sama sobre tormentas y granizos, y los de Lorente sobre sequías.

Los economistas, por su parte, han dedicado sus trabajos al estudio de los daños causados en la agricultura. Poseen como antecedente los trabajos de Pigou de 1920, en los que trataba la incidencia que tenían los sucesos atmosféricos extremos en las actividades productivas.

No obstante, no existe un gran volumen de trabajos sobre este tema, ya que, para la realización de este tipo de estudios es necesario abarcar periodos de años muy largos de los que no se posee una información sistemática ni cuantitativa. Así, los primeros registros instrumentales en España se remontan a 1737 y son series muy cortas y sin continuidad. Este hecho nos lleva a recurrir a la información procedente de fuentes indirectas, que es mucho más difícil de tratar y su fiabilidad es mucho menor.

Existen estudios que tratan este tema, utilizando diferentes fuentes y métodos de trabajo para su confección. Así M. Barriendos Vallvé en «La climatología histórica en el estudio de los riesgos climáticos. El episodio de noviembre de 1617 en Catalunya», distingue tres tipos de fuentes documentales: eclesiásticas, municipales y privadas. Dentro de éstas, los documentos que suelen contener la información climática utilizable son los libros de actas, libros de memoria o dietarios. Cabe mencionar, los estudios basados en rogativas llevados a cabo por J. Martín Vide y M. Barriendos Vallvé.

La prensa también esta siendo utilizada como fuente para estudios climáticos. Así, E. Duce Díaz ha realizado un estudio sobre los efectos de los fenómenos meteorológicos sobre la agricultura en España entre 1985-1990, utilizando básicamente en su elaboración el compendio total de números del diario La Vanguardia. En la misma línea, A. P. Pérez García-Torres en su trabajo «Avenidas fluviales e inundaciones en el Medio Vinalopó en el tránsito de los siglos XIX y $X X »$, también utiliza la prensa como una de sus fuentes de estudio.

Otros autores, que en los últimos años han abordado el estudio de los acontecimientos extraordinarios son J. Olcina Cantos, A. Rico Amorós y A. Gil Olcina, que han centrado sus investigaciones en el Levante español. Por su parte, M. F. Pita López, también ha contribuido al estudio del clima histórico mediante sus trabajos sobre el cambio climático.

\section{Objetivo y fuentes}

El objetivo de la investigación consiste en la realización de un estudio de los acontecimientos climáticos extraordinarios acaecidos en el periodo comprendido entre 1870 y 1954 
de las tres provincias que componen la Comunidad Autónoma Vasca. Concretamente, este informe pretende:

- Crear un listado histórico de cada fenómeno objeto de estudio.

- Estudio de cada fenómeno realizando una comparación entre las tres provincias.

- Caracterización de cada provincia y de la Comunidad Autónoma del País Vasco (C.A.P.V.) según los fenómenos climáticos extraordinarios.

Para la confección de este estudio las fuentes utilizadas han sido la base de datos KLIMAT XX, para la cual se utiliza el programa Knosys, y la bibliografía existente sobre el tema de estudio.

No obstante, la fuente principal ha sido la base de datos. Ésta consiste en la recopilación de artículos de prensa de contenido climático, recogidos en una serie de periódicos diarios de la C.A.P.V. Los periódicos utilizados son diferentes según la provincia a la que se refiera.

- En Álava

El porvenir alavés

Semanario católico

El Gorbea

La libertad

La voz del pueblo

Diario de Álava

Heraldo Alavés

El pensamiento alavés

El alavés

El anunciador vitoriano

- En Bizkaia
Villa de Bilbao
Eco vascongado
Euscalduna
Eco Bilbaino
El Nervión
La gaceta del norte
El porvenir vascongado
El noticiero bilbaino
El liberal
El pueblo vasco
Euzkadi
La tarde
Hierro

- En Gipuzkoa

Diario de San Sebastián

La paz

El Urumea

Eco de San Sebastián

La voz de Guipúzcoa

El fuerista

La libertad

La unión vascongada

El correo de Guipúzcoa

La constancia

El pueblo vasco

El correo del norte

El diario vasco

El País Vasco

El día

La voz de España

Cada periódico pertenece a un periodo de tiempo determinado. Algunos de ellos se solapan entre sí, por lo que para algunas épocas tenemos información de varios periódicos. Sin embargo, en algunos años, concretamente en el siglo XIX, nos encontramos con vacíos de información.

Las noticias recopiladas en la prensa son implementadas en fichas que están estructuradas en varios campos:
1. Fecha
2. Periódico
3. Texto
4. Identificadores
5. Temperatura máxima
6. Temperatura mínima
7. Temperatura media
8. Precipitación
9. Observatorio
10. Provincia
11. Localización 
El campo «identificadores» es clave para la búsqueda de la información en la base de datos ya que mediante éste, se codifican las noticias. Los identificadores que se utilizan son los siguientes:

$\begin{array}{llll}\text { Lluvia } & \text { Nieve } & \text { Ciclón } & \text { Rogativas } \\ \text { Aguacero } & \text { Rayo } & \text { Crecida } & \text { Calor1, Calor2 } \\ \text { Temporal de lluvia } & \text { Viento1 } & \text { Inundación } & \text { Frío1, Frío2 } \\ \text { Granizo } & \text { Vendaval } & \text { Sequía } & \text { Helada } \\ \text { Boletín } & \text { Daños } & \text { Oleaje } & \text { Astronomía } \\ \text { Valores } & \text { - Materiales } & \text { Marítimo } & \text { Pronóstico } \\ \text { - Temperatura } & \text { - Humanos } & \text { Marea } & \text { Augurios } \\ \text { - Presión } & \text { - Sociales } & \text { Volcán } & \text { Lúdico } \\ \text { - Viento } & \text { Salud } & \text { Seísmo } & \text { Resumen } \\ \text { - Precipitación } & \text { Agrario } & \text { Desprendimiento } & \text { Extra } \\ \text { Niebla } & \text { Fauna } & \text { Geología } & \text { Rutina } \\ \text { Deshielo } & \text { Ecología } & \text { Innovación } & \text { Otros } \\ \text { Cambio } & \text { Incendio } & \text { Anécdota } & \end{array}$

Mediante la combinación de estos identificadores entre sí y con los restantes campos se pueden realizar múltiples búsquedas según la información que interese. A continuación se adjunta un ejemplo:

Se desea conocer si se han producido nevadas de carácter extraordinario durante el año 1888 en la provincia de Álava. Para ello, se rellenan los campos de los que poseemos información y el programa realiza una selección de las noticias que cumplen las condiciones especificadas.

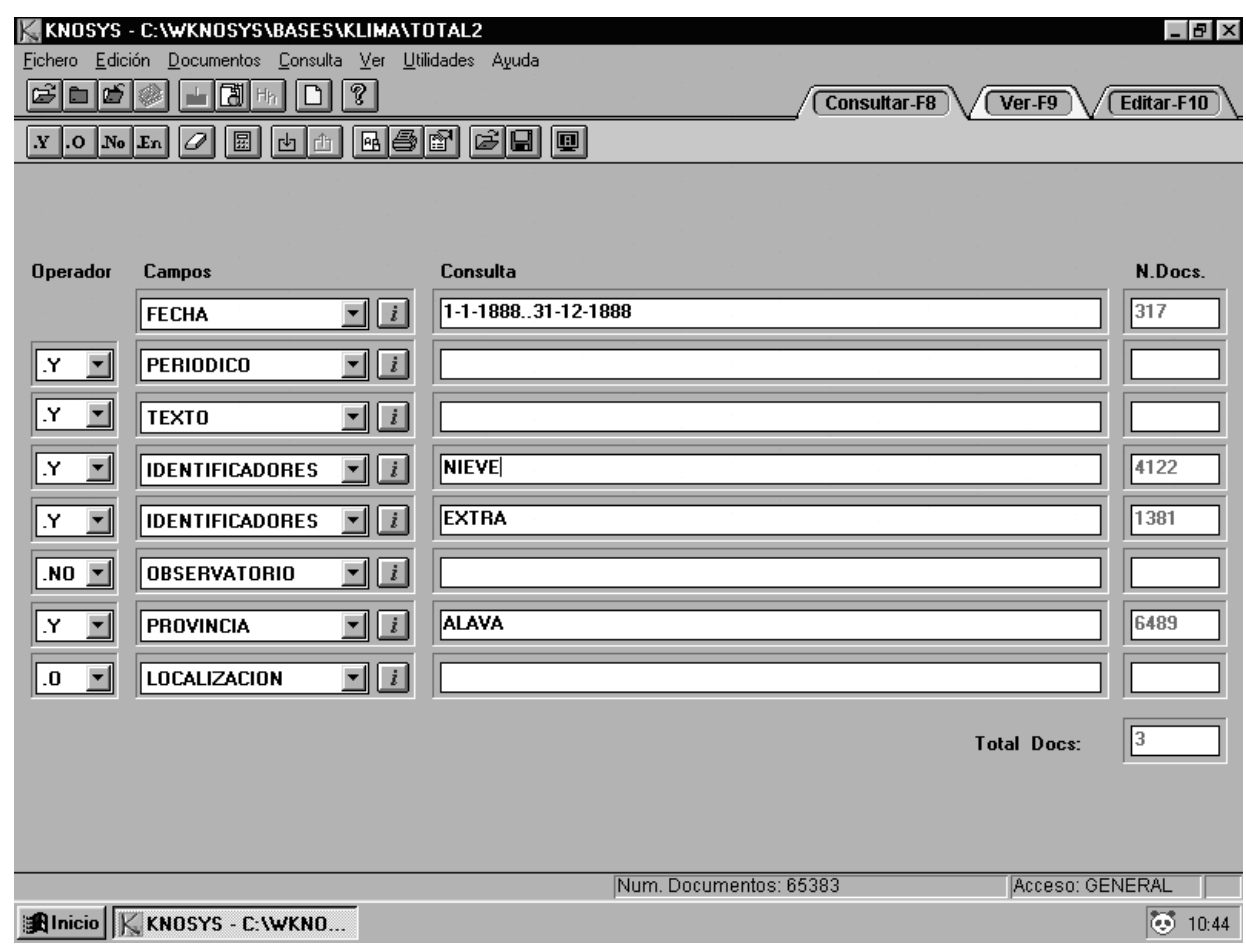

Fuente: Base de datos KLIMAT XX. 
En este caso, la base de datos ha encontrado tres noticias, de las que a continuación, se muestra una de ellas.

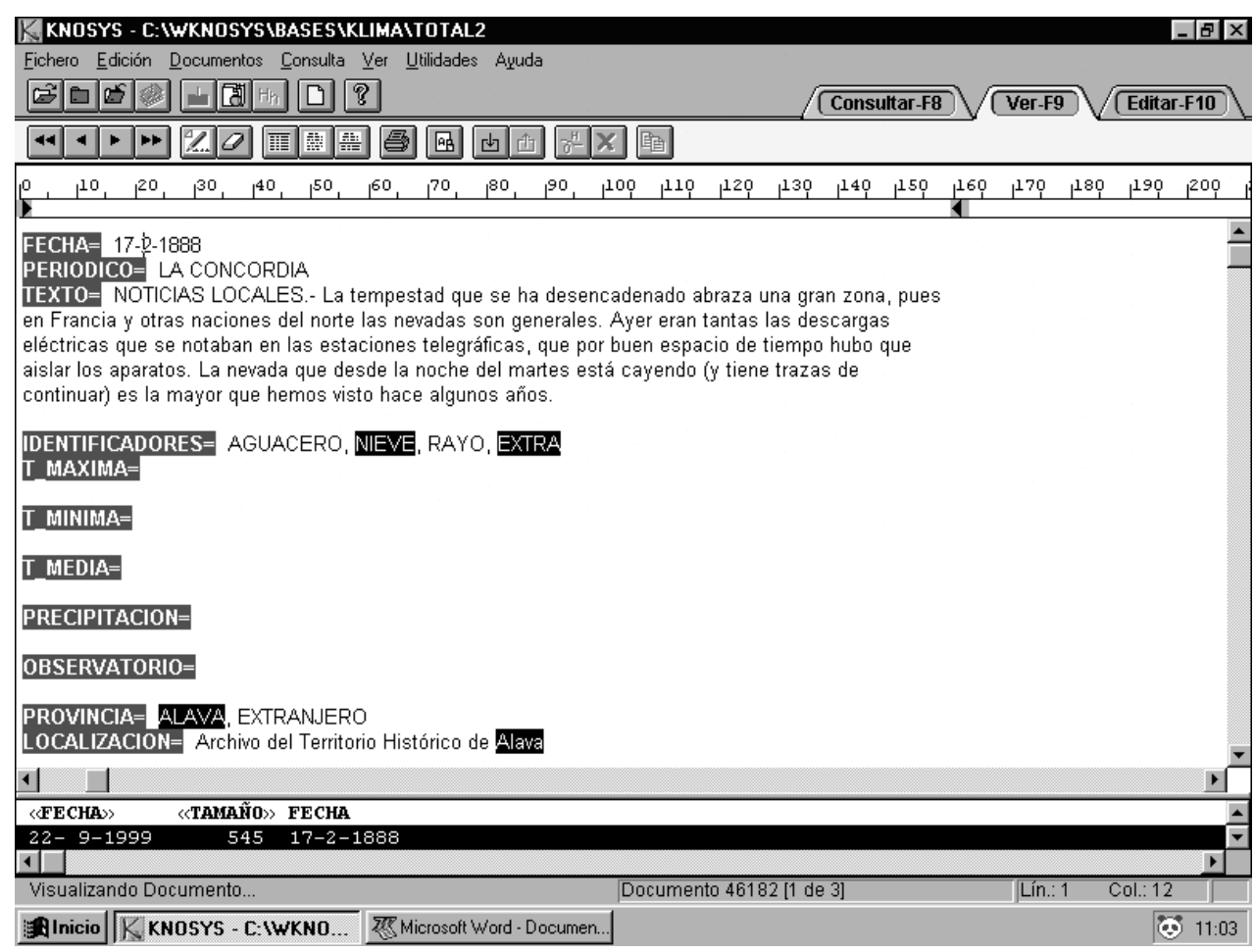

Fuente: Base de datos KLIMAT XX.

\section{Metodología}

La metodología seguida se puede resumir en las siguientes fases:

1. Selección del periodo de estudio.

El periodo elegido para esta investigación corresponde a los años que van de 1870 a 1954, debido a que la fuente principal — Base de Datos KLIMAT XX — posee suficiente información de las tres provincias a estudio — Álava, Bizkaia y Gipuzkoa— para ese periodo de tiempo.

2. Selección de los fenómenos a estudio.

Para elegir los fenómenos a estudio, se ha llevado a cabo un primer filtrado, en el que se han cruzado todos los identificadores que se corresponden con los fenómenos, que en ocasiones, pueden dar lugar a acontecimientos extraordinarios y suponer un riesgo climático, con el identificador «extra». Este identificador se incluye en aquellas noticias que narran acontecimientos especiales que pueden catalogarse de extraordinarios debido a su intensidad o a su rara frecuencia.

De esta manera, se han seleccionado los fenómenos de los que se posee suficiente información en la base de datos para la realización de su análisis: 
-Aguacero

-Temporal de lluvia

-Crecida

-Inundación

-Granizo
-Nieve

-Helada

-Frío

-Calor

-Sequía
-Rayo

-Vendaval

-Temporal de mar

3. Lectura de las noticias

Una vez conseguida esta información se ha procedido a seleccionar mediante una lectura rápida aquella que realmente era valiosa para nuestra investigación. De esta manera, se han escogido solamente las noticias correspondientes a las tres provincias vascas y se han desechado aquellas que no hemos considerado extraordinarias (debido a que el identificador «extra» no se refería al fenómeno en cuestión).

Para organizar y resumir la información de las noticias seleccionadas, se ha rellenado una ficha, previamente diseñada, para cada acontecimiento, con los datos más significativos. Todas las noticias de un mismo acontecimiento han sido agrupadas en cada ficha.

\begin{tabular}{|c|c|c|c|}
\hline \multicolumn{4}{|l|}{ FICHA DE ACONTECIMIENTO } \\
\hline FENÓMENO & & & \\
\hline PROVINCIA & ÁLAVA & BIZKAIA & GIPUZKOA \\
\hline FECHA / PERIODO & & & \\
\hline $\mathrm{N}^{\circ}$ DE NOTICIAS & & & \\
\hline DAÑOS MATERIALES & & & \\
\hline DAÑOS HUMANOS & & & \\
\hline DAÑOS SOCIALES & & & \\
\hline IDENTIFICADORES ASOCIADOS & & & \\
\hline VAL. NUMÉRICOS & & & \\
\hline NOTAS & & & \\
\hline
\end{tabular}

Elaboración propia

Esta fase ha constituido la mayor inversión de tiempo, debido al gran volumen de la información extraída tras el primer filtrado.

4. Elaboración de listas de fenómenos extraordinarios

Con la documentación definitiva se ha elaborado un listado con la fecha de la ocurrencia de los episodios de cada uno de los fenómenos, o en su defecto, de la fecha de la noticia en que quedaron reflejados.

5. Realización de gráficas

A partir de este listado se ha procedido a la consecución de una serie de gráficas, tras la reducción de la información a valores cuantitativos, en la medida de lo posible. Con ello, se ha efectuado un análisis de la distribución mensual y evolución histórica de cada fenómeno. 
6. Análisis de cada fenómeno por provincias y comparación de las mismas

Se ha realizado el análisis por provincias a partir de las listas de fenómenos extraordinarios y de las gráficas elaboradas, comentando su distribución mensual, su evolución histórica, los daños que ocasionan y aquellos eventos más significativos.

7. Caracterización de cada una de las provincias y de la C.A.P.V según los acontecimientos extraordinarios

Se ha realizado una gráfica - resumen de los fenómenos extraordinarios, a partir de la cual se ha llevado a cabo una caracterización de cada una de las provincias y de la C.A.P.V atendiendo a los acontecimientos extraordinarios más importantes a las que están sujetas. Posteriormente tras la realización de una gráfica de la evolución histórica, se han comentado los periodos que más sucesos han registrado.

\section{Dificultades encontradas}

Los problemas surgidos a la hora de abordar este estudio han sido los siguientes:

— Los derivados de la información contenida en la base de datos. Por un lado, encontramos vacíos de información en los siguientes periodos de tiempo: en Álava de 1875 a 1879 y de 1884 a 1886, en Bizkaia de 1870 a 1874 y en Gipuzkoa de 1870 a 1872. Además, el número de periódicos revisados para cada provincia es diferente. Así, para el caso de Álava son 10, para Bizkaia 13 y para Gipuzkoa son 16. A todo esto hay que añadir, que las series de que consta cada periódico, son más largas para el caso de Gipuzkoa, y más cortas las de Álava. Todo esto supone que la cantidad de información que se posee para cada provincia varíe en su volumen.

- La inexactitud de los identificadores. La dificultad de restringir el significado de cada identificador provoca que la asignación de los mismos a las noticias suponga un cierto grado de subjetividad.

- La fiabilidad de la información recogida en los periódicos. Cabe reseñar que esta información es de tipo subjetivo y su veracidad depende de la rigurosidad del informador.

- La dificultad de transformar la información cualitativa en cuantitativa. Este inconveniente es significativo en aquellos fenómenos que no se suelen producir de forma puntual, como sequías, olas de frío, etc., ya que es prácticamente imposible establecer el principio y fin del periodo de ocurrencia.

- La inexactitud de las fechas en que se producen los acontecimientos. En muchas ocasiones en las noticias no se registra la fecha en que ha acontecido el fenómeno, por lo que se le ha tenido que asignar una fecha aproximada, como la fecha del periódico, o la fecha en la que se escribe la noticia.

\section{Los acontecimientos extraordinarios en nuestro estudio}

En la base de datos KLIMAT XX, se ha codificado con el identificador «extra» aquellas noticias en que aparecen acontecimientos que de alguna forma pueden ser considerados especiales, debido a la rarísima frecuencia con la que se producen o a la intensidad del fenómeno.

Dada la dificultad de definir de manera objetiva el umbral mediante el cual un episodio puede ser clasificado como extraordinario sólo han quedado catalogadas aquellas situaciones en las que el periodista, por propia consideración o tomando la opinión popular, 
califica una situación como excepcional. Así, el que las personas mayores del lugar manifestaran que nunca habían conocido un acontecimiento semejante, es un indicador para considerar este hecho como extraordinario. Aunque, hay que tener en cuenta, que la memoria climática de las personas no es muy fiable, ya que está condicionada por un alto grado de subjetividad.

Bajo este identificador, por tanto, se pueden considerar como acontecimientos extraordinarios una temperatura alcanzada en un momento dado, una gran inundación, una nevada intensa,...

\section{Caracterización de las provincias según los fenómenos extraordinarios}

\section{1. Álava}

Tabla 1

FENÓMENOS EXTRAORDINARIOS EN ÁLAVA

\begin{tabular}{|l|c|c|}
\hline FENÓMENO & $\mathbf{N}^{\mathbf{0}}$ ACONTECIMIENTOS & $\mathbf{N}^{\mathbf{0}}$ NOTICIAS \\
\hline Nieve & 92 & 101 \\
\hline Rayo & 38 & 72 \\
\hline Frío & $37^{*}$ & 85 \\
\hline Temporal de lluvia & 33 & 62 \\
\hline Aguacero & 25 & 40 \\
\hline Inundación & 24 & 47 \\
\hline Calor & $23^{*}$ & 29 \\
\hline Granizo & 19 & 21 \\
\hline Crecida & 18 & 35 \\
\hline Vendaval & 11 & 11 \\
\hline Sequía & $10^{*}$ & 23 \\
\hline
\end{tabular}

FUENTE: Base de datos KLIMAT XX. Elaboración propia.

* Hace referencia al $\mathrm{n}^{\circ}$ de periodos en que ocurrió el acontecimiento (el resto hace referencia al $\mathrm{n}^{\circ}$ de días).

Si observamos la tabla de acontecimientos extraordinarios referente a la provincia de Álava, podemos ver que los fenómenos relacionados con las bajas temperaturas son los que mayor relevancia poseen. En este sentido, podemos decir que los fenómenos más característicos de esta provincia son el frío, las heladas y las nevadas.

Así, en el periodo 1870-1954 se han registrado en la prensa 37 periodos de frío, reflejados en 85 noticias. En los casos más extremos el termómetro ha llegado a marcar temperaturas inferiores a $-20^{\circ} \mathrm{C}$. Pero en general, la temperatura «habitual» en las olas de frío ha variado entre los 10 y 20 grados bajo cero. La razón de la existencia de estas olas de frío se debe a dos factores: la situación geográfica y la topografía. A pesar de que la provincia no se encuentra muy lejos en línea recta de la costa, las características del relieve la convierten en una zona de interior, de forma que la influencia del mar sobre las temperaturas queda muy atenuada.

Unido a este fenómeno, se encuentra el de la helada, incluido en muchas ocasiones en los periodos de frío. Éstas son ocasionadas por la masa polar marítima, la ártica marítima y la polar 
continental. Debido al carácter eminentemente agrícola de Álava, las heladas han supuesto grandes pérdidas económicas, ya que han afectado negativamente a cosechas y pastos.

Otro evento característico son las grandes nevadas. En el periodo de estudio se han contabilizado 92 días de nieve, los cuales en su mayoría se produjeron en los meses de invierno. Generalmente, este fenómeno está asociado a gotas frías o flujos del norte fríos y húmedos, unido a una depresión localizada en el Mediterráneo occidental procedente de Escandinavia. Por otro lado, se pueden apreciar desemejanzas dentro de esta región, ocasionadas por el factor altitud. Así, las zonas más elevadas poseen mayor número de días de nevada y las intensidades son mayores.

Además de los fenómenos citados anteriormente, cabe destacar el de la sequía. En la época de estudio se han registrado 10 periodos en los cuáles ha existido escasez de agua. Su importancia radica en su repercusión en la economía de la región, especialmente, dentro del sector primario. A la hora de hablar de los factores que influyen en el descenso de la pluviosidad, cabe destacar la topografía. La disposición E - W de la divisoria cantábrico mediterránea supone una barrera a las masas de aire húmedas procedentes del Cantábrico. Las masas de aire al toparse con dicho obstáculo, sufren una desecación que provoca una disminución de las precipitaciones en Álava. Este hecho implica, que también existan menos aguaceros y temporales, lo que a su vez supone un menor número de crecidas e inundaciones.

\subsection{Bizkaia}

Tabla 2

FENÓMENOS EXTRAORDINARIOS EN BIZKAIA

\begin{tabular}{|l|c|c|}
\hline FENÓMENO & $\mathbf{N}^{\mathbf{0}}$ ACONTECIMIENTOS & $\mathbf{N}^{\mathbf{0}}$ NOTICIAS \\
\hline Nieve & 109 & 119 \\
\hline Temporal de lluvia & 85 & 175 \\
\hline Vendaval & 69 & 76 \\
\hline Rayo & 67 & 127 \\
\hline Frío & $65^{*}$ & 117 \\
\hline Aguacero & 56 & 101 \\
\hline Inundación & 45 & 129 \\
\hline Crecida & 45 & 108 \\
\hline Calor & $43^{*}$ & 73 \\
\hline Temporal de mar & $41^{*}$ & 72 \\
\hline Granizo & 28 & 40 \\
\hline Sequía & $18^{*}$ & 33 \\
\hline
\end{tabular}

FUENTE: Base de datos KLIMAT XX. Elaboración propia.

*Hace referencia $a l n^{\circ}$ de periodos en que ocurrió el acontecimiento (el resto hace referencia al $n^{\circ}$ de días).

En Bizkaia, los fenómenos más característicos son los relacionados con las precipitaciones intensas. Es decir, el temporal, la crecida y la inundación.

En el caso de los temporales, el número de acontecimientos registrados por la prensa, ha sido de 85 , recogidos en un total de 175 noticias. Con estos primeros datos, podemos observar cómo se trata de un fenómeno al que la prensa presta mucha atención. Este hincapié puede ser debido a su relación con posibles inundaciones y/o 
crecidas, lo que provoca a su vez, cuantiosos daños materiales y la alteración de la vida cotidiana.

La mayoría de los temporales se producen en los meses invernales. La causa de la existencia de estos temporales es debido a dos situaciones: a la causada por la corriente del NW en altura que trae consigo aire polar marítimo; y a la ocasionada por la trayectoria zonal de la circulación del W.

Una consecuencia de los temporales, es la crecida del caudal de los cursos de agua. En esta provincia, se han recogido 45 crecidas, registradas en 108 noticias. Al igual, que en el caso de los temporales, los periódicos han prestado bastante interés a estos acontecimientos por sus posibles consecuencias. Las causas principales para que se sucedan las crecidas están ligadas a la pluviosidad - fuertes precipitaciones - y a las características de la hidrografía atlántica —ríos cortos, de fuertes pendientes y con valles muy antropizados-.

Como consecuencia de temporales y crecidas, nos encontramos con las inundaciones. El número de acontecimientos recogidos por la prensa es de 45 en 129 noticias. En números relativos, es el acontecimiento más comentado por la prensa. La explicación de su interés viene marcado por las consecuencias económicas y humanas que traen consigo.

Las inundaciones que tienen lugar a finales de otoño, invierno e inicio de primavera se deben a los temporales, mientras que las inundaciones de finales de primavera y de verano e inicio del otoño, suelen estar ligadas a aguaceros.

Observando la tabla, podemos señalar cómo la nieve posee el mayor número de acontecimientos en esta provincia, 109 acontecimientos en 119 noticias. Sin embargo, no consideramos que por ello, sea el fenómeno de mayor importancia, ya que la mayoría se dan en zonas de altitud elevada y su consecuencia principal, es el corte de vías de comunicación.

\subsection{Gipuzkoa}

Tabla 3

FENÓMENOS EXTRAORDINARIOS EN GIPUZKOA

\begin{tabular}{|l|c|c|}
\hline FENÓMENO & $\mathbf{N}^{\mathbf{0}}$ ACONTECIMIENTOS & $\mathbf{N}^{\mathbf{o}}$ NOTICIAS \\
\hline Temporal de lluvia & 101 & 231 \\
\hline Vendaval & 100 & 130 \\
\hline Nieve & 99 & 121 \\
\hline Frío & $65^{*}$ & 126 \\
\hline Temporal de mar & $63^{*}$ & 92 \\
\hline Rayo & 63 & 124 \\
\hline Calor & $61^{*}$ & 83 \\
\hline Inundación & 53 & 169 \\
\hline Aguacero & 53 & 101 \\
\hline Crecida & 35 & 98 \\
\hline Granizo & 28 & 46 \\
\hline Sequía & $24^{*}$ & 41 \\
\hline
\end{tabular}

FUENTE: Base de datos KLIMAT XX. Elaboración propia.

*Hace referencia al $n^{\circ}$ de periodos en que ocurrió el acontecimiento (el resto hace referencia al $n^{\circ}$ de días)

En la provincia de Gipuzkoa, los fenómenos más característicos son el temporal de lluvia, el vendaval, el temporal de mar y el calor. 
En cuanto al temporal de lluvia, se han registrado en esta región 101 acontecimientos en 231 noticias. Las causas que originan los temporales y las consecuencias que provocan en la actividad humana son similares a las de la otra provincia costera.

Respecto al vendaval, podemos observar en la tabla que el número de acontecimientos recogidos son 100, en 130 noticias. Generalmente, los fuertes vientos que se dan en esta provincia poseen la dirección $\mathrm{N}$ y NW y en menor medida dirección S. Éstos últimos son los de mayor velocidad y los que más daños ocasionan. Este es un viento típico de la costa vasca característico de la estación otoñal. Se trata de un viento cálido procedente del sur peninsular, que tras atravesar la Cordillera Cantábrica, en su descenso se calienta y deseca, provocando este mismo efecto a las tierras que recorre.

Un porcentaje significativo de este número de vendavales corresponde a las galernas. Este es un fenómeno propio de la costa cantábrica, sobre todo de la zona oriental. La galerna es un fenómeno ampliamente recogido por la prensa debido a las graves consecuencias que conlleva, tanto materiales como humanas, en los pueblos costeros.

Una situación relacionada con el vendaval es el temporal de mar. Se han registrado 63 periodos de temporal de mar, reflejados en 92 noticias. La importancia de esto radica en la influencia que posee en la actividad pesquera, así como en los daños materiales y humanos que ocasionan.

Otro fenómeno a tener en cuenta en esta provincia es el calor. Encontramos 61 periodos, reflejados en 83 noticias. Principalmente, estos episodios de calor se deben a la acción del viento sur, que trae consigo masas de aire cálido, que debido al efecto föehn provocan una intensificación del calor y una desecación de las tierras a las que llega. Una de las consecuencias más importantes de las olas de calor es el agudizamiento de la sequía, que puede afectar a la vida cotidiana de los habitantes de la zona debido a los cortes del suministro de agua y al funcionamiento normal de las industrias.

\section{Fenómenos extraordinarios en la C.A.P.V. (1870 - 1954)}

\subsection{Caracterización de la C.A.P.V. según los fenómenos extraordinarios}

Tabla 4

FENÓMENOS EXTRAORDINARIOS EN LA C.A.P.V SEGÚN $N^{\circ}$ DE ACONTECIMIENTOS

\begin{tabular}{|c|c|c|c|}
\hline ALAVA & BIZKAIA & GIPUZKOA & C.A.P.V. \\
\hline Nieve (92) & Nieve (109) & Temporal de lluvia (101) & Nieve (300) \\
\hline Rayo (38) & Temporal de lluvia (85) & Vendaval (100) & Temporal de lluvia (219) \\
\hline Frío (37) * & Vendaval (69) & Nieve (99) & Vendaval (180) \\
\hline Temporal de lluvia (33) & Rayo (67) & Frío $(65) *$ & Rayo (168) \\
\hline Aguacero (25) & Frío $(65) *$ & Temporal de mar (63) * & Frío (167) * \\
\hline Inundación (24) & Aguacero (56) & Rayo (63) & Aguacero (134) \\
\hline Calor $(23)^{*}$ & Inundación (45) & Calor (61) * & Calor (127) * \\
\hline Granizo (19) & Crecida (45) & Inundación (53) & Inundación (122) \\
\hline Crecida (18) & Calor $(43) *$ & Aguacero (53) & Temporal de mar (104) * \\
\hline Vendaval (11) & Temporal de mar $(41)^{*}$ & Crecida (35) & Crecida (98) \\
\hline \multirow[t]{2}{*}{ Sequía $(10)^{*}$} & Granizo (28) & Granizo (28) & Granizo (75) \\
\hline & Sequía (18) * & Sequía $(24) *$ & Sequía (52) * \\
\hline$($ Total $=329)$ & $($ Total $=671)$ & $($ Total $=745)$ & (Total $=1745)$ \\
\hline
\end{tabular}

FUENTE: Base de datos KLIMAT XX. Elaboración propia.

* Hace referencia al $\mathrm{n}^{\circ}$ de periodos en que ocurrió el acontecimiento (el resto hace referencia al $\mathrm{n}^{\circ}$ de días) 


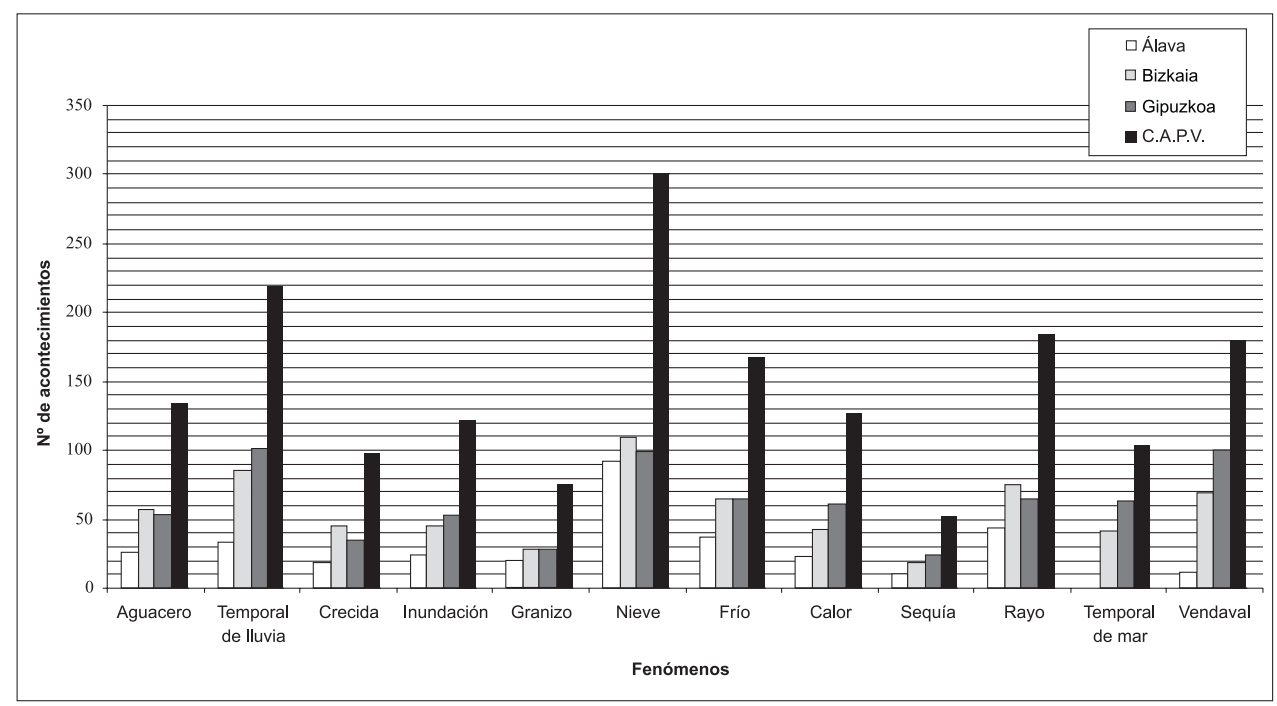

GRÁFICA 1. Fenómenos meteorológicos extraordinarios (1870-1954)

FUENTE: Base de datos KLIMAT XX. Elaboración propia.

A la hora de valorar los resultados que se obtienen al observar la tabla precedente, hay que tener en cuenta que el análisis de los fenómenos en unos casos se ha realizado por periodos y en otros por días. Este aspecto provoca que la comparación cuantitativa pueda llevar a error. Además, no hay que olvidar, que el criterio seguido para catalogar con el identificador «extra» a las noticias de frío y de nieve ha sido diferente dependiendo de la provincia a la que se refiriera. Así, en Álava, se han considerado extraordinarias las nevadas de gran magnitud, mientras que en las provincias litorales, el carácter excepcional se debe a su inusualidad.

El total de acontecimientos registrados por la prensa en el País Vasco para el periodo de estudio (1870-1954) ha sido de 1745, de los cuales Gipuzkoa representa el 42'6\%, Bizkaia el 38'5\% y Álava el 18'9\%. Estos datos muestran que las provincias litorales son las responsables de la mayor parte de los acontecimientos extraordinarios de esta comunidad autónoma.

La cantidad de noticias referidas a un acontecimiento extraordinario está en función de la gravedad de las consecuencias que provoca. Generalmente, el periodista dedica la mayor parte de la noticia a describir los daños que ocasiona el evento. La relación de daños que comentamos en este apartado es una extracción de la información que se refleja en las mismas.

En la C.A.P.V. la nieve es el fenómeno que más días ha registrado noticias, con 109 en Bizkaia, 99 en Gipuzkoa y 92 en Álava. A priori, Álava debería ser la provincia con un mayor número de acontecimientos, pero por los motivos comentados anteriormente, las provincias costeras le superan.

La nieve produce daños en el sector agrario, especialmente en Álava; dificulta las comunicaciones, provocando, por un lado, la incomunicación de algunos pueblos, y por otro lado, accidentes de tráfico y sus consiguientes perjuicios para la vida humana.

En segundo lugar se sitúa el temporal de lluvia, con 219 días en que se ha recogido noticias, siendo Gipuzkoa la provincia que más días aglutina, con un $46 \%$ del total, mientras que Álava sólo supone un $15 \%$. 
Los daños ocasionados son cuantiosos, ya que dan lugar a graves inundaciones tanto en los campos como en las zonas urbanas; así como desprendimientos que interrumpen la circulación por carretera y ferrocarril.

A continuación se encuentra el vendaval, con 180 días registrados. Dentro de éste se incluyen las galernas que se producen en las provincias costeras. La magnitud del valor total se debe sobre todo a la provincia de Gipuzkoa, al contabilizar un 55\% de los acontecimientos. En Álava la importancia de este fenómeno es bastante pequeña, con tan sólo 11 fenómenos acaecidos.

Los vendavales provocan numerosos daños en el mobiliario urbano, en las edificaciones y en los parques y jardines así como la dificultad de tránsito de los viandantes, llegando a provocar en ocasiones caídas. Además, al ser un fenómeno estrechamente vinculado a los temporales de mar, cabe destacar los problemas que ocasionan en la actividad pesquera, en el tráfico marítimo y en las embarcaciones. Algunos acontecimientos provocaron la muerte de numerosos pescadores.

Los sucesos relacionados con rayos ocupan la cuarta posición en esta «jerarquía» de acontecimientos extraordinarios, con un total de 168, siendo Bizkaia la que más reúne (67). Aunque Álava posee el menor número de eventos de rayo en la C.A.P.V., dentro de la provincia es el segundo fenómeno más frecuente (38).

Los daños que causan los rayos son cuantiosos, tanto materiales como humanos. Para realizar este estudio sólo se han tenido en cuenta los últimos, contabilizándose 34 muertos en Álava, más de 48 en Bizkaia y más de 61 en Gipuzkoa. Además de más de 17, 46 y 31 heridos respectivamente. La mayor parte de este tipo de sucesos tienen lugar en el ámbito rural, afectando principalmente al sexo masculino.

\subsection{Evolución histórica de los acontecimientos extraordinarios}

El estudio de la evolución histórica de los acontecimientos extraordinarios en la C.A.P.V. de 1870 a 1954, muestra dos periodos significativos. El primero transcurre de 1895 a 1917 y el segundo de 1924 a 1935.

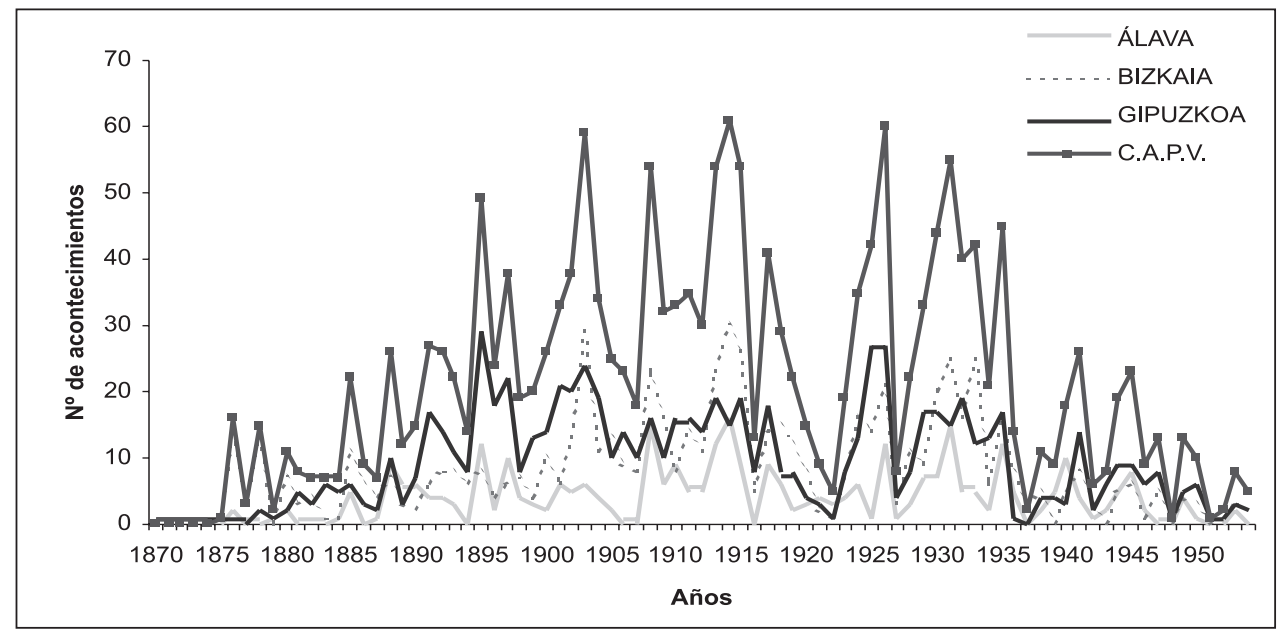

GRÁFICA 2. Evolución histórica del no de acontecimientos extraordinarios (1870-1954)

FUENTE: Base de datos KLIMAT XX. Elaboración propia. 
De 1895 a 1917 se han contabilizado 812 acontecimientos excepcionales, los cuales suponen un 46'1\% del total. Dentro de este lapso de tiempo destacan los siguientes años:

1895 A Los acontecimientos extraordinarios constatados han sido 49, con 12 en Álava, 8 en Bizkaia y 29 en Gipuzkoa. Destacan los fenómenos relacionados con la precipitación, principalmente la nieve en Álava y Gipuzkoa y los temporales de lluvia en Bizkaia y Gipuzkoa. También cabe mencionar el vendaval en Gipuzkoa.

1903 A En este año se han registrado 59 acontecimientos extraordinarios, 29 en Bizkaia, 24 en Gipuzkoa y 6 en Álava. Ocupa el tercer lugar en cuanto al número de acontecimientos registrados en nuestro periodo de estudio. Esto se debe sobre todo a aguaceros y temporales de lluvia y de mar.

1908 A Se han contabilizado 54 acontecimientos extraordinarios, 15 en Álava, 23 en Bizkaia y 16 en Gipuzkoa. Los fenómenos que más se han repetido han sido la inundación, la crecida y el temporal de lluvia en Álava y Bizkaia; y el temporal de mar y el vendaval en Gipuzkoa.

1913 A Se han recogido 54 acontecimientos extraordinarios, 12 en Álava, 23 en Bizkaia y 19 en Gipuzkoa. El fenómeno más destacable en las tres provincias es la sequía, con 13 periodos de sequía excepcionales. También son reseñables el granizo y el temporal de lluvia en Bizkaia y el rayo en Gipuzkoa.

1914 A Este año, con 61 acontecimientos, es el de mayor registro de todo el periodo de estudio. Álava posee 16, Bizkaia 30 y Gipuzkoa 15. La nieve es el fenómeno más significativo en las tres provincias, con 17 días de nevadas extraordinarias. También cabe destacar el temporal de lluvia en Bizkaia y las sequías y los sucesos relacionados con el rayo en Gipuzkoa.

1915 A Este año se han registrado 54 acontecimientos extraordinarios, con 9 en Álava, 26 en Bizkaia y 19 en Gipuzkoa. Las tres provincias se vieron afectadas por la mayor parte de los fenómenos estudiados, aunque podemos señalar que en las provincias litorales el vendaval y la sequía son los fenómenos más frecuentes.

1917 A Se han contabilizado 41 acontecimientos extraordinarios, con 9 en Álava, 14 en Bizkaia y 18 en Gipuzkoa. En las tres provincias destaca el fenómeno de la nieve, sumando 15 nevadas en total. Además, en Gipuzkoa se produjeron 6 días de granizo.

El segundo periodo corresponde a los años que van de 1924 a 1935. En estos doce años el número de acontecimientos acaecidos ha sido de 447 , lo que supone un $25 \% 3 \%$ del total. Cabe destacar los siguientes años:

1925 A Los acontecimientos extraordinarios constatados han sido 42, con 1 en Álava, 14 en Bizkaia y 27 en Gipuzkoa. Las provincias litorales son las responsables de este valor, sobresaliendo los fenómenos relacionados con la precipitación (temporal de lluvia, inundación y nieve).

1926 A Este año ocupa el segundo lugar en la «jerarquía» de acontecimientos extraordinarios en nuestro periodo de estudio, con 60. Álava posee 12, Bizkaia 21 y Gipuzkoa 27. En las tres provincias el fenómeno más frecuente es la nieve con 17 días de nevadas excepcionales. Además en Bizkaia son destacables los fenómenos relacionados con las precipitaciones intensas; y en Gipuzkoa los periodos de calor y sequía y los vendavales.

1930 A Se han recogido 44 acontecimientos extraordinarios, con 7 en Álava, 20 en Bizkaia y 17 en Gipuzkoa. Los fenómenos más repetidos en las tres provincias son los relacionados con la precipitación, sumando un total de 23 eventos. En Bizkaia también se puede citar el rayo y en Gipuzkoa el vendaval y la sequía. 
1931 A Se han contabilizado 55 acontecimientos extraordinarios, con 15 en Álava, 25 en Bizkaia y 15 en Gipuzkoa. La nieve es el fenómeno más frecuente, con un total de 17 días, aunque podemos mencionar los periodos de frío en Álava y Gipuzkoa; y los periodos de sequía en Bizkaia y Gipuzkoa,

1935 A Se han registrado 45 acontecimientos extraordinarios, con 12 en Álava, 16 en Bizkaia y 17 en Gipuzkoa. El 55\% de los mismos corresponden a las grandes nevadas que sufrieron las tres provincias. A éstas hay que unir los 7 periodos de frío que sufrió todo el territorio.

En resumen, en los dos periodos comentados, los acontecimientos extraordinarios se registran sobre todo en las provincias litorales, en especial en Gipuzkoa. Los fenómenos meteorológicos que más se repiten son, principalmente, las nevadas extraordinarias y los relacionados con las fuertes precipitaciones (aguaceros, temporal de lluvia, crecida e inundación).

\section{Conclusiones}

1. Tratamiento del clima por parte de la prensa

En este estudio, referente a los acontecimientos extraordinarios ocurridos en el País Vasco de 1870 a 1954, se ha podido apreciar que en la prensa se le da mayor importancia a las consecuencias que provoca el acontecimiento extraordinario que al propio fenómeno climático en sí mismo. Así, cuanto más graves son los daños ocasionados, mayor es el número de noticias en que se refleja. En este sentido, puede ocurrir que acontecimientos extraordinarios que no hayan ocasionado daños de importancia en la actividad humana, no se mencionen o se traten de forma superficial.

2. Validez de la prensa como fuente de estudio para investigaciones climáticas

Hemos podido observar que a través de la información obtenida de la prensa se puede llegar a tener una idea aproximada de como ha sido el clima en un periodo de estudio determinado. Mediante la consulta de bibliografía, se ha comprobado que las conclusiones obtenidas para nuestro periodo de estudio, se asemejan bastante a las dadas por otros autores.

Con todo esto podemos afirmar que las fuentes indirectas, especialmente para épocas preinstrumentales, tienen un gran valor a la hora de realizar un estudio climático.

3. Dificultad de encontrar periodicidad en la sucesión de eventos excepcionales

Desde épocas remotas, la humanidad ha intentado buscar ciclos que rigieran el comportamiento del clima. El interés por controlar las consecuencias, incluso catastróficas, que este tipo de sucesos ocasionan ha sido un factor determinante para promover esta clase de estudios. No obstante, la necesidad de largos periodos de datos y la complejidad de estos estudios provoca que no sea una tarea fácil.

En nuestro caso, hemos podido observar que, según los resultados obtenidos para nuestro periodo de estudio, los acontecimientos meteorológicos extraordinarios no están sujetos a ninguna periodicidad claramente definida.

\section{Bibliografía}

ALMARZA MATA C. (2000) «Variaciones climáticas en España. Época instrumental» en «El Campo», $n^{\circ}$ 137. Ed. Servicios de estudios BBVA, pp. 69-87.

AREITIO PIEDRA J., MUÑOZ SANZ P., TORRIJO MURCIANO R. y JIMÉNEZ MUNTEAGUDO J. L.(1995) «Las galernas del Cantábrico: Un riesgo poco frecuente» en Situaciones de riesgo climático en España. Instituto Pirenaico de Ecología, Jaca (Huesca). Ed. José Creus Novau, pp. 197-209. 
BARRIENDOS VALLVÉ, M. (1995) «La climatología histórica en el estudio de los riesgos climáticos. El episodio de noviembre de 1617 en Catalunya» en Situaciones de riesgo climático en España. Instituto Pirenaico de Ecología, Jaca (Huesca). Ed. José Creus Novau, pp. 73-85.

GALDOS URRUTIA, R. (1995) «La transición climática en la provincia de Alava» en Ibaiak eta Haranak. Ed. Etor.

GIL OLCINA, A. et all (1986) Inundaciones en la Ciudad y Término de Alicante. Instituto Universitario de Geografía y Ayuntamiento de Alicante. Alicante.

LÓPEZ GÓMEZ, A. (1956) «Las heladas de febrero de 1956 en Valencia» en Estudios Geográficos, $n^{\circ}$ 65. Madrid, pp. 673-696.

LORENTE, J. M. (1945) «La sequía del invierno 1944-45 en España» en Revista de Geofísica. IV. Madrid. 1945.

MARCOS VALIENTE, O. (2001) «Sequía: definiciones, tipologías y métodos de cuantificación» en Investigaciones Geográficas, $\mathrm{n}^{\circ}$ 26. Instituto Universitario de Geografía, Universidad de Alicante, pp. 59-81.

MARTÍN VIDE, J. Y BARRIENDOS VALLVÉ, M. (2000) «El clima del pasado: la perspectiva histórica» en El Campo, No 137. Ed. Servicios de estudios BBVA, pp. 49-69.

MARTÍN VIDE, J. y OLCINA CANTOS, J. (2001) Climas y tiempos de España. Ed. Alianza Editorial. 258 pp.

MEAZA RODRÍGUEZ, G. y RUIZ URRESTARAZU, E. (dir.) (1996) Euskal Herriko Geografia. Klima eta Urak, Ostoa S.A., Lasarte-Oria.

MORALES GIL, A. OLCINA CANTOS, J. y RICO AMORÓS, A. M. (2000) «Diferentes percepciones de la sequía en España: adaptación, catastrofismo e intentos de corrección» en Investigaciones Geográficas. no 23, pp. 5-47.

OLCINA CANTOS, J. (1994) Riesgos climáticos en la Península Ibérica. Ed. Acción Divulgativa, S.L. 440 pp.

OLCINA CANTOS, J. (1995) «El factor climático y la ordenación del territorio. Los riesgos climáticos» en Situaciones de riesgo climático en España. Instituto Pirenaico de Ecología, Jaca (Huesca). Ed. José Creus Novau. Pp. 13-73.

PÉREZ GARCÍA-TORRES, A. P. (2001) «Avenidas fluviales e inundaciones en el Medio Vinalopó en el tránsito de los siglos XIX y XX» en Investigaciones Geográficas, $\mathrm{n}^{\circ}$ 26. Instituto Universitario de Geografía, Universidad de Alicante, pp. 169-181.

PITA LÓPEZ, M.F. (2001): «Sequías en la cuenca del Guadalquivir». En Causas y consecuencias de las sequías en España. Coords./Eds.: Gil Olcina, A. y Morales Gil, A. pp. 303-344. Instituto Universitario de Geografía y Caja de Ahorros del Mediterráneo.

RUIZ URRESTARAZU, E. y GALDOS URRUTIA, R. (1989) «El clima de la vertiente atlántica del País Vasco», en Ibaiak eta Haranak. Ed. Etor.

RUIZ URRESTARAZU, E. (dir.) (1998) El clima del País Vasco a través de la prensa. Ed. Servicio Vasco de Meteorología del Gobierno Vasco. 211 pp.

SAMA, N. (1925) «Tormentas y granizadas» en Catecismos del Agricultor y Ganaderos, $\mathrm{n}^{\circ}$ 86. Ed. Espasa- Calpe. Madrid. Pp. 23-31.

URIARTE, A. (1983) «Régimen de precipitaciones en la costa $N W$ y $N$ de la Península Ibérica». Tesis Doctoral, Caja de Guipúzcoa.

VILAR BONET, F. (1995) «Situaciones, riesgos y recurrencia de tormentas intensas en el Pirineo Oriental» en Situaciones de riesgo climático en España. Instituto Pirenaico de Ecología, Jaca (Huesca). Ed. José Creus Novau. Pp. 105-117.

\section{Diccionarios geográficos}

COLIAS GIL, J. (Coord.) (1996) Gran Vox. Diccionario de términos de Geografía. Ed. Bibliograf, S.A. Barcelona.

FONTANILLO MERINO, E. (y colaboradores) (1986) Diccionario de Geografía. Ed. E.G. Anaya S.A. Madrid.

WHITTOW, J. B. (1984) Diccionario de Geografía Física. Ed. Cast. Alianza Editorial, S.A. Madrid. 